\title{
Studies of Electric Dipole Moment and Magnetic Dipole Moment of Substituted Benzothiazolyl and Benzimidazolyl Derivatives
}

\author{
ANKUSH W. WAKODE ${ }^{1 *}$; ARCHANA S. BURGHATE ${ }^{2}$ and SHRIKANT A. WADHAL ${ }^{3}$ \\ Department Of Chemistry, Shri Shivaji Science College, Amravati, 444603,(M.S.) India. \\ ${ }^{*}$ Corresponding author E-mail: ankushwakode @ gmail.com \\ http://dx.doi.org/10.13005/ojc/330319
}

(Received: March 24, 2017; Accepted: May 13, 2017)

\begin{abstract}
Refractive indices of benzothiazolyl and benzimidazolyl derivatives with different concentration in binary liquid mixture such as acetone-water, Dioxane-water \& DMSO-water at $35 \pm 0.1{ }^{\circ} \mathrm{C}$ were measured by Abbe's refractometer. The data obtained was utilized to calculate molar polarization \& electric dipole moment which explain solute-solvent interactions. The magnetic susceptibility and magnetic dipole moment calculated by using Gouy's method.
\end{abstract}

Keywords: Electric dipole moment, Effective magnetic moment, Magnetic susceptibility.

\section{INTRODUCTION}

Benzothiazolyl and benzimidazolyl substituted derivatives are the heterocyclic compounds with wide application in pharmaceutical chemistry and have wide range of biological applicability ${ }^{1}$. In the present work, Benzothiazolyl and benzimidazolyl derivatives are widely found in bioorganic and medicinal chemistry with applications in drug discovery and development for treatment such as autoimmune and inflammatory diseases in the prevention of solid organ transplant rejection, epilepsy $^{2}$, antitumor, antiviral ${ }^{3}$, anticonvulsant ${ }^{4}$, antiallergic, antitumor ${ }^{5}$, neuroprotective and immunosuppressive properties. The studies of refractive indices are being increasingly used as tools $s^{6.7}$ for investigation of the physical properties of pure components and the nature of inter molecular interaction between the liquid mixture constituents ${ }^{8}$ Refractive index measurements in combination with density, boiling point, melting point and other analytical data have wide applicability in chemical analysis an industry ${ }^{9}$ Many researchers studied Schiff's bases with the determination of physical parameter ${ }^{10}$. Schiff's bases uses are some of the most widely used organic compound they are use as pigment and dyes, catalysts, intermediates in organic synthesis, and as polymer stabilizers. Analytical performance of refractometry in quantitative Estimation of isotopic concentration of heavy water in nuclear reactor ${ }^{11}$.Many workers ${ }^{12-13}$ studied physical parameters of organic compounds binary liquid mixtures to judge molecular interactions. 
Measurement of magnetic properties has been used to characterize a wide range of systems from oxygen, metallic alloys, solid state materials, and coordination complexes containing metals. Most organic and main group element compounds have all the electrons paired and these are diamagnetic molecules with very small magnetic moments. All materials show some reaction to a magnetic field though is the case of conventionally non-magnetic materials the reaction will be very weak a powerful electromagnet and sensitive measuring instrument are needed to demonstrate these weak reactions. The magnetic properties like magnetic susceptibility, magnetic moment can be studied from Gouy method. In present study electric dipole moment is determined from refractometric study and magnetic dipole moment by Gouy's method.

\section{EXPERIMENTAL}

Acetone-water, Dioxane-water and DMSOwater mixture as well as solutions of Benzothiazolyl and benzimidazolyl substituted derivatives of different concentration were prepared. All weighing was made on contech electronic balance $( \pm 0.001 \mathrm{~g})$. The accuracy of density measurements was within $0.1 \mathrm{~kg} \mathrm{~m}^{-3}$. The refractive indices of solvent mixture and solutions were measured by Abbes' refractometer was within $( \pm 0.001)$. The densities of the solutions were determined by pyknometer. The temperature of prism box was maintained at $36^{\circ} \mathrm{C}$. Initially, the refractometer was calibrated with glass piece ( $n=1.5220$ ) provided with the instrument. The magnetic measurement was carried out by Gouy's method and $\mathrm{CuSO}_{4} \cdot 5 \mathrm{H}_{2} \mathrm{O}$ was used as calibrant.

The present work deals with the study of molar refraction and polarizability constant of
Benzothiazolyl and benzimidazolyl substituted derivatives in different concentration of binary mixture at $307 \mathrm{~K}\left(35^{\circ} \mathrm{C}\right)$. The data obtained have been used to compute intermolecular interaction. The selected compounds for the study may be summarised as

The various equations used in electric and magnetic dipole moment study may be reported as

$$
\begin{aligned}
& R_{m}=\frac{\left(n^{2}-1\right)}{\left(n^{2}+2\right)}\left\{\frac{\left[x_{1} m_{1}+x_{2} m_{2}+x_{3} m_{3}\right]}{d}\right\} \\
& R=-\pi N_{Q} \alpha \\
& \mathrm{P}_{\mathrm{o}}=\mathrm{R}_{2}=\frac{\left(n^{2}-1\right)}{\left(n^{2}+2\right)} \times \frac{M_{2}}{d} \\
& \mu=0.128 \times 10^{-18} \times\left(\mathrm{P}_{2} \mathrm{~T}\right)^{1 / 2} \text { debye } \\
& \mu=\frac{3 \mathrm{KT} \chi_{\mathrm{m}}}{\text { No }} \\
& \chi_{\mathrm{g}}=\frac{2 m g}{A \mu_{0} H^{2}} \\
& \mu_{\text {eff }}=2.828\left(\chi_{\text {AT }}\right)^{1 / 2} \\
& \text { RESULTS AND DISCUSSION }
\end{aligned}
$$

Refractive index is an important physical parameter of liquids. The potential of refractometry depends on sensitivity and time of measuring. In the present study refractive index of solvent-water mixture and Benzothiazolyl and benzimidazolyl substituted derivatives in solvent water mixture were determined. Molar refraction and polarizability electric dipole moment were determined for variation

\begin{tabular}{lcc}
\hline Name of compounds & Symbol & Molecular formula \\
\hline $\begin{array}{l}\text { 1-Benzothiazole-2-yl-[1,2]diazetidin-3-one } \\
\text { (1-Benzothiazol-2-yl-[1,2]diazetidine-3-ylidene) }\end{array}$ & $1 \mathrm{a}$ & $\mathrm{C}_{9} \mathrm{H}_{7} \mathrm{~N}_{3} \mathrm{SO}$ \\
$\begin{array}{l}\text {-4(phenyl-thiazole-2-yl)-amine } \\
\text { 1-Benzothiazol-2-I-[1,2]diazetidine-3-ylidene) }\end{array}$ & $1 \mathrm{~b}$ & $\mathrm{C}_{18} \mathrm{H}_{13} \mathrm{~N}_{5} \mathrm{~S}_{2}$ \\
$\begin{array}{l}\text {-phenyl-amine } \\
\text { 1,2-Dihydro-benzo[4,5]imidazo[2,1-c] }\end{array}$ & $1 \mathrm{C}$ & $\mathrm{C}_{15} \mathrm{H}_{12} \mathrm{~N}_{4} \mathrm{~S}$ \\
$\begin{array}{l}\text { [1,2,4]triazin-3-one } \\
\text { (1,2-Dihydro-benz[4,5]imidazo[2,1-c][1,2,4]triazin-3-ylidene) }\end{array}$ & $2 \mathrm{~b}$ & $\mathrm{C}_{9} \mathrm{H}_{8} \mathrm{~N}_{4} \mathrm{O}$ \\
-(4-phenyl-thiazole-2-yl)-amine & & $\mathrm{C}_{18} \mathrm{H}_{14} \mathrm{~N}_{6} \mathrm{~S}$ \\
\hline
\end{tabular}


Table1.1: Molar Polarization and electric dipole moment w.r.t. concentration variation

\begin{tabular}{|c|c|c|c|c|}
\hline \multicolumn{2}{|c|}{ System: $1 \mathrm{a}$ in $75 \%$} & \multirow{2}{*}{$\begin{array}{c}\text { Dioxane-water } \\
\alpha \times 10^{-23}\end{array}$} & \multicolumn{2}{|c|}{ Temperature: $35 \pm 0.1 \mathrm{C}$} \\
\hline Sr.No. & CONC. (M) & & $\mathbf{P}_{\mathrm{o}}$ & $\mu($ esu-cm) \\
\hline 1 & 0.01 & 0.006873 & 0.173274 & $9.35088 \mathrm{E}-19$ \\
\hline 2 & 0.005 & 0.008556 & 0.215706 & $1.04332 \mathrm{E}-18$ \\
\hline 3 & 0.0025 & 0.01016 & 0.256147 & $1.13692 \mathrm{E}-18$ \\
\hline 4 & 0.00125 & 0.01201 & 0.302809 & $1.23615 \mathrm{E}-18$ \\
\hline 5 & 0.000625 & 0.014162 & 0.35705 & 1.3423E-18 \\
\hline
\end{tabular}

Table1.2: Molar Polarization and electric dipole moment w.r.t. concentration variation

System: $2 a$ in $75 \%$ Dioxane-water Temperature: $35 \pm 0.1 \mathrm{C}$

\begin{tabular}{ccccc}
\hline Sr.No. & CONC. (M) & $\alpha \times 10^{-23}$ & $\mathbf{P}_{\circ}$ & $\boldsymbol{\mu}(\mathbf{e s u}-\mathrm{cm})$ \\
\hline 1 & 0.01 & 0.012308 & 0.310304 & $1.25135 \mathrm{E}-18$ \\
2 & 0.005 & 0.013684 & 0.344995 & $1.31945 \mathrm{E}-18$ \\
3 & 0.0025 & 0.01379 & 0.347686 & $1.32458 \mathrm{E}-18$ \\
4 & 0.00125 & 0.013945 & 0.351578 & $1.33197 \mathrm{E}-18$ \\
5 & 0.000625 & 0.014306 & 0.360696 & $1.34914 \mathrm{E}-18$ \\
\hline
\end{tabular}

Table 1.3: Molar Polarization and electric dipole moment w.r.t. concentration variation

System: 1C in 75\% Dioxane-water Temperature: $35 \pm 0.1 \mathrm{C}$

\begin{tabular}{ccccc}
\hline Sr.No. & CONC. (M) & $\alpha \times 10^{-23}$ & $\mathbf{P}_{\circ}$ & $\boldsymbol{\mu}(\mathbf{e s u}-\mathrm{cm})$ \\
\hline 1 & 0.01 & 0.00769 & 0.193885 & $9.8914 \mathrm{E}-19$ \\
2 & 0.005 & 0.00774 & 0.19514 & $9.92335 \mathrm{E}-19$ \\
3 & 0.0025 & 0.007785 & 0.196275 & $9.95218 \mathrm{E}-19$ \\
4 & 0.00125 & 0.008731 & 0.220125 & $1.05395 \mathrm{E}-18$ \\
5 & 0.000625 & 0.010042 & 0.253191 & $1.13034 \mathrm{E}-18$ \\
\hline
\end{tabular}

Table 1.4: Molar Polarization and electric dipole moment w.r.t. concentration variation

System: $1 \mathrm{a}$ in $75 \% \quad$ Acetone-water Temperature: $35 \pm 0.1 \mathrm{C}$

\begin{tabular}{ccccc}
\hline Sr.No. & CONC. (M) & $\alpha \times$ 10-23 $^{-23}$ & $\mathbf{P}_{\circ}$ & $\boldsymbol{\mu}$ (esu-cm) \\
\hline 1 & 0.01 & 0.076164 & 1.92025 & $3.11289 \mathrm{E}-18$ \\
2 & 0.005 & 0.105864 & 2.669065 & $3.66999 \mathrm{E}-18$ \\
3 & 0.0025 & 0.106959 & 2.696664 & $3.68892 \mathrm{E}-18$ \\
4 & 0.00125 & 0.108923 & 2.746186 & $3.72263 \mathrm{E}-18$ \\
5 & 0.000625 & 0.10999 & 2.773091 & $3.74083 \mathrm{E}-18$ \\
\hline
\end{tabular}


Table 1.5: Molar Polarization and electric dipole moment w.r.t. concentration variation

System: $2 \mathrm{a}$ in $75 \% \quad$ Acetone-water Temperature: $35 \pm 0.1 \mathrm{C}$

\begin{tabular}{ccccc}
\hline Sr.No. & CONC. (M) & $\alpha \times 10^{-23}$ & $\mathbf{P}_{\circ}$ & $\boldsymbol{\mu}($ esu-cm) \\
\hline 1 & 0.01 & 0.155084 & 3.90999 & $4.44194 \mathrm{E}-18$ \\
2 & 0.005 & 0.164555 & 4.148791 & $4.57558 \mathrm{E}-18$ \\
3 & 0.0025 & 0.161438 & 4.070202 & $4.53204 \mathrm{E}-18$ \\
4 & 0.00125 & 0.18447 & 4.650881 & $4.84454 \mathrm{E}-18$ \\
5 & 0.000625 & 0.192787 & 4.860568 & $4.95255 \mathrm{E}-18$ \\
\hline
\end{tabular}

Table 1.6: Molar Polarization and electric dipole moment w.r.t. concentration variation

System: 1c in 75\% Acetone-water Temperature: $35 \pm 0.1 \mathrm{C}$

\begin{tabular}{ccccc}
\hline Sr.No. & CONC. (M) & $\alpha \times 10^{-23}$ & $\mathbf{P}_{\circ}$ & $\boldsymbol{\mu}(\mathbf{e s u}-\mathbf{c m})$ \\
\hline 1 & 0.01 & 0.102004 & 2.57174 & $3.60246 \mathrm{E}-18$ \\
2 & 0.005 & 0.105057 & 2.648719 & $3.65598 \mathrm{E}-18$ \\
3 & 0.0025 & 0.107465 & 2.709412 & $3.69762 \mathrm{E}-18$ \\
4 & 0.00125 & 0.108666 & 2.739689 & $3.71823 \mathrm{E}-18$ \\
5 & 0.000625 & 0.111973 & 2.823069 & $3.77438 \mathrm{E}-18$ \\
\hline
\end{tabular}

Table 1.7: Molar Polarization and electric dipole moment w.r.t. concentration variation

System: 1a in 75\% DMSO-water Temperature: $35 \pm 0.1 \mathrm{C}$

\begin{tabular}{ccccc}
\hline Sr.No. & CONC. (M) & $\alpha \times 10^{-23}$ & $\mathbf{P}_{\circ}$ & $\boldsymbol{\mu}$ (esu-cm) \\
\hline 1 & 0.01 & 0.03246 & 0.818372 & $2.03 \mathrm{E}-18$ \\
2 & 0.005 & 0.039128 & 0.986494 & $2.23 \mathrm{E}-18$ \\
3 & 0.0025 & 0.04352 & 1.097226 & $2.35 \mathrm{E}-18$ \\
4 & 0.00125 & 0.048497 & 1.222721 & $2.48 \mathrm{E}-18$ \\
5 & 0.000625 & 0.051282 & 1.292926 & $2.55 \mathrm{E}-18$ \\
\hline
\end{tabular}

Table 1.8: Molar Polarization and electric dipole moment w.r.t. concentration variation

System: $2 a$ in 75\% DMSO-water Temperature: $35 \pm 0.1 \mathrm{C}$

\begin{tabular}{ccccc}
\hline Sr.No. & CONC. (M) & $\alpha \times \mathbf{1 0}^{-23}$ & $\mathbf{P}_{\text {o }}$ & $\boldsymbol{\mu}(\mathbf{e s u}-\mathrm{cm})$ \\
\hline 1 & 0.01 & 0.00096 & 0.024211 & $3.5 \mathrm{E}-19$ \\
2 & 0.005 & 0.002764 & 0.069698 & $5.93 \mathrm{E}-19$ \\
3 & 0.0025 & 0.00398 & 0.100349 & $7.12 \mathrm{E}-19$ \\
4 & 0.00125 & 0.006274 & 0.158175 & $8.93 \mathrm{E}-19$ \\
5 & 0.000625 & 0.007388 & 0.186279 & $9.7 \mathrm{E}-19$ \\
\hline
\end{tabular}


Table 1.9: Molar Polarization and electric dipole moment w.r.t. concentration variation

System: $1 \mathrm{c}$ in $75 \% \quad$ DMSO-water Temperature: $35 \pm 0.1 \mathrm{C}$

\begin{tabular}{ccccc}
\hline Sr.No. & CONC. (M) & $\alpha \times 10^{-23}$ & $\mathbf{P}_{\circ}$ & $\boldsymbol{\mu}$ (esu-cm) \\
\hline 1 & 0.01 & 0.041096 & 1.036116 & $2.29 \mathrm{E}-18$ \\
2 & 0.005 & 0.041881 & 1.055898 & $2.31 \mathrm{E}-18$ \\
3 & 0.0025 & 0.050713 & 1.278585 & $2.54 \mathrm{E}-18$ \\
4 & 0.00125 & 0.050866 & 1.28244 & $2.54 \mathrm{E}-18$ \\
5 & 0.000625 & 0.057625 & 1.452841 & $2.71 \mathrm{E}-18$ \\
\hline
\end{tabular}

Table-2

\begin{tabular}{lcccc}
\hline $\begin{array}{l}\text { Sr } \\
\text { No. }\end{array}$ & $\begin{array}{c}\text { Name of } \\
\text { System }\end{array}$ & Mol. Wt. & $\begin{array}{c}\chi_{\mathbf{m}\left(\mathbf{m}^{3} \mathbf{r}\right.} \\
\mathbf{m o l}^{-1)}\end{array}$ & $\boldsymbol{\mu}_{\text {eff }}(\mathrm{BM})$ \\
\hline 1 & $2 \mathrm{a}$ & 188.19 & 0.020557 & 0.223193 \\
2 & $1 \mathrm{~b}$ & 363.47 & 0.045376 & 0.331599 \\
3 & $1 \mathrm{c}$ & 280.36 & 0.035 & 0.291231 \\
4 & $1 \mathrm{a}$ & 205.25 & 0.025624 & 0.249184 \\
5 & $2 \mathrm{~b}$ & 346.42 & 0.043247 & 0.323728 \\
6 & $1 \mathrm{e}$ & 325.36 & 0.040618 & 0.313734 \\
\hline
\end{tabular}

in concentration of compounds in $75 \%$ solvent-water mixture. The respective polarizability and electric dipole moment may be presented as in table-1.

In the present investigation, with decrease in concentration of compounds, polarizability as well as electric dipole moment increases in order of solvent mixture Acetone-water > DMSO-water > Dioxan-water from the results it may be predicted that for the compounds $1 \mathrm{a}, 2 \mathrm{a}, 1 \mathrm{c}$ in various solvents, results in increase in polarity this results due to solvent-solvent interaction is greater than solutesolvent interaction.

The some compounds were implemented for magnetic properties study by using Gouy method.
Solids exhibiting only electronic polarizability are elemental solid dielectrics. Solids which manifest electronic and ionic polarizabilities are ionic nonpolar dielectrics and solids which possess orientational, electronic and ionic polarizabilities are polar solids.

In the present study for the compounds $1 \mathrm{a}, 1 \mathrm{~b}, 1 \mathrm{c}, 2 \mathrm{a}, 2 \mathrm{~b}$ shows specific value for magnetic dipole moment. The existence of magnetic property in $1 b, 1 c$, and $2 b$ may be due to dipole formation at aliphatic ring across $>\mathrm{C}=\mathrm{N}$ - group where as in case of $1 \mathrm{a}$ and $2 \mathrm{a}$, the dipole formation may be at carbonyl group. These dipoles may be formed due to electronegativity difference in the binding atoms. The observed values of magnetic susceptibility and magnetic dipole moment in the substituted benzothiazolyl and benzimidazolyl compounds predicts that their magnetic nature may be due to spin and orbital motion of electrons ${ }^{14}$.

\section{ACKNOWLEDGEMENT}

The authors would like to thanks authorities of Shri Shivaji Science College, Amravati University, Amravati for providing necessary facilities.

\section{REFERENCES}

1. Wakode, A. W.; Burghate, A. S.; Wadhal,S. A.Indo American Journal of Pharmaceutical Research .2014, 4, 5010-5016.

2. Hangirgekar, S. P.; Shirodkar, S. G.Indo American Journal of Pharmaceutical Research. 2011, 1, 153-157.
3. Kawde, P.R.; Deohate, P. P.; Berad B. N. Indian Journal of Chemistry.2015,54B, 833-836.

4. Singh, M.; Gangwar, M.; Nath, G.; Singh, S. K. Indian Journal of Chemistry.2014,52, 10621070.

5. Baheti, K. G.; Jadhav, J. S.; Suryavanshi, A. 
T. Indian Journal of Chemistry.2005,44B, 834-837.

6. Nain, A. K.; Chandra, P.; Pandey, J.D.; Gopal,S. Journal of Chemical \& Engineering Data.2008, 53, 2654-2665.

7. Nain, A. K.. Fluid Phase Equilibria, 2008, 265, 56.

8. Ansari, N. H.Open Journal of Physical chemistry,2014,4,1-5.

9. Ubarhande, S. S.; Burghate, A. S.;Berad, B.N.; Turak,J.D. Rasayan J. Chem. 2011, 4, 585587.
10. Talegaonkar, ${ }^{*}$ R. S.; Burghate, A. S.; Wadhal, S. A.Indian J. Heterocyclic Chem. Vol.2011,20, 413-414.

11. Dhole,K.; Ghosh, S.; Datta, A.; Tripathy, M. K.; Bose, H.Barc Newsletter,2011, 320, 7-11.

12. Wakode,A.W.; Burghate, A. S.; Wadhal,S.A. RasayanJ. Chem.2016,4, 386-392.

13. Talegaonkar, R. S.; Burghate, A. S; Wadhal, S. A. Orient. J. Chem. 2011, 27, 1285-1288. Pillai S. O. Solid State Physics. Seventh edition, New Age International $(P)$ Limited, Publication. 2015. 